\title{
La Documentacion y el nacimiento de las Naciones Unidas
}

\section{Félix Sagredo Fernández}

Facultad de Ciencias de la Información

Universidad Complutense de Madrid (España)

\subsection{Resumen}

Se examina el compromiso internacionalista de Paul Otlet en la Unión de Asociaciones Internacinales y se pone en relación con el carácter progresista y positivista del proyecto científico de la Documentación frente a sus precedentes históricos.

Palabras clave: Otlet, Paul. Unión de Asocaciones Internacionales. Documentación. Historia.

\subsection{Abstract}

The internationalist commitment of Paul Otlet in the Union of International Associations is examined and put in relation with the progressive and positivist character of the Documentation Science scientific project.

Keywords: Otlet, Paul. Union of International Associations. Documentation. History.

\section{Introducción}

A finales del mes de mayo de 1995, me encontraba en la Universidad de California, campus de Berkeley, a donde había llegado con una Beca del Amo concedida por la Universidad Complutense de Madrid. Sobre mi mesa de trabajo de la School of Library and Information Science - que ese año cambio su nombre a School of Information Mangement and Systems -, me encontré una mañana el semanario Berkeleyan (1995). En primera plana aparecían los motivos del momento informativo: una noticia sobre la visita del Secretario de Trabajo del Gobierno norteamericano, Robert Reich, por aquel entonces; la presentación del programa informático DARS que mejoraría a partir de 1995-1996 los servicios de información de la Universidad; y un artículo de Fernando Quintero (1995), sobre el anciano impresor de 81 años del Campus de Berkeley, Joe Baxley. Puesto que 
este último artículo trataba temas de la edición en Berkeley a mediados de los 40, especialmente en dicho campus, me puse a leerlo; y, cuál fue mi sorpresa al encontrar en él ciertas connivencias con un tema que, desde que había encontrado en la Biblioteca Real Alberto I de Bruselas el tratado de Constitución Mundial de Paul Otlet (1917), me rondaba en la cabeza.

Conocidas son las ideas internacionalistas de los primeros documentalistas belgas, fraguadas en la creación del Mundaneum (Union of International Associations, 1928; Mundaneum..., s. f.), que finalmente ha recuperado el reposo documental e investigador en el número 76 de la Rue du Nimy, en la ciudad de Mons - precisamente, la ciudad belga del libro, después de un azaroso recorrido por los barrios e instituciones bruselenses desde el primer tercio del pasado siglo. Por otra parte, el artículo en cuestión comenzaba de una forma muy apropiada para un documentalista, en plena era de la revolución informático-documental, mediados los 90 del pasado siglo, y en los términos siguientes: "Gone are the bulky linotype machines and cylinder presses that occupied the first floor of UC Printing Building on Oxford and Center streets. Modern press technology has replaced them with computers [...]". Joe Baxley, relataba en el texto, cincuenta años después, los avatares de la preparación de la edición —en sólo cuatro días y en cinco idiomas, a saber, inglés, francés, español, ruso y chino- de la Carta de las Naciones Unidas, que había de ser presentada y firmada por cincuenta países a finales de junio de aquel año, concluidas las jornadas, cercanas al ocaso de la II Guerra Mundial, de la célebre Conferencia de San Francisco. Los textos en los tres primeros idiomas fueron relativamente fáciles de componer; los tipos estaban al alcance de cualquier impresor. Los precisos para la redacción en ruso del texto, de los que sólo fue localizado un ejemplar en todo San Francisco - es decir un modelo de tipo en caracteres cirílicos - fue algo muy trabajoso. En lo referente al chino, la edición hubo de confiarse a una imprenta de Chinatown; si bien se utilizó el sistema de impresión de izquierda a derecha, y de arriba abajo, como en los otros cuatro idiomas, en vez de derecha a izquierda y de abajo arriba, como es preceptivo en el idioma chino. Baxley vivió los momentos más estresantes de su vida, sobre todo al darse cuenta de que en la edición definitiva, y a última hora, faltaba un cuadernillo de cuatro páginas. Como característica de la edición, adoptaron para la versión inglesa un formato similar al de la Biblia de Gutenberg, de 1454, editada en Maguncia, con el fin de conseguir que las 145 páginas de texto tuvieran el mismo número de líneas que el histórico incunable bíblico gutenbergiano. La carta debía de estar a las diez de la mañana junto al Monumento a la Guerra, aquel día 26 de junio de 1945, donde se procedería a su rúbrica por los representantes de los cincuenta estados presentes. "Sólo nos demoramos diez minutos en su entrega", comentaba Baxley en la citada entrevista. Los impresores de la Carta, en esos precisos momentos se encontraban en sus casas durmiendo

Scire. $10: 1$ (en.-jun. 2004) 21-30. 
y recuperándose de una angustiosa experiencia de noventa horas de trabajo en la imprenta en tan sólo cuatro días. "We never got any credit", concluye Baxley.

Quizás más de uno de ustedes se pregunte a qué viene esta curiosa introducción. No se preocupen que voy a justificar el título de la breve exposición que les presento.

\section{La Sociedad de Naciones y la Organización de las Naciones Unidas}

Todos conocemos por la Historia la tristísima realidad bélica de la pasada centuria: dos guerras mundiales, 1914-1918 y 1939-1945, que después de asolar grandes regiones del planeta, dejaron en la sociedad mundial varias decenas de millones de muertos; un poso de infinita amargura en las sociedades contemporáneas, un miedo insuperable que cristalizó para más angustia en casi cuarenta años de Guerra Fría; y una fuente de discordias internacionales indescriptible; adversidades no concluidas aún en nuestros días, y con las que convivimos algunos de los aquí presentes hasta la caída del Muro de Berlín en 1989.

Uno de los intelectuales más tocado y sensibilizado por los terribles efectos del azote bíblico fue el creador de la ciencias bibliológica y documental a finales del siglo XIX y primera mitad del XX, Paul Marie Ghislain Otlet. Su inseparable compañero de inquietudes y fatigas Henry La Fontaine recibiría incluso uno de los primeros Nobel de la Paz, precisamente en 1913. Para entonces, la Unión de Asociaciones Internacionales, que presidía el eximio documentalista, había publicado numerosas obras de Paul Otlet al respecto, realizadas solo o en colaboración: L'Organisation Internationale et les Associations Internationales (1909), La Vie Internationale et l'effort pour son Organisation (1912), La fin de la Guerre: Traité de Paix Général basé sur Charte Mondiale déclarant les droits de L'Humanité et organisant la Confédération des Etats (1914) y Les Problèmes internationaux et la Guerre (1916). Todos los tratadistas de Derecho Internacional y autores de obras sobre historia e internacionalismo en el siglo XX, reconocen que la denominada Constitución Mundial fue la madre del texto de la Carta editada en San Francisco y adoptada por la ONU a partir de octubre del mismo año (Cot y Pellet, 1991; Simma, 1995; Charte..., 1947). Se le dio el nombre de Carta - soslayando el de Tratado, Convención o similar- para reflejar el deseo de mensaje profundo a una sociedad harta ya de tratados postbélicos, que poco después eran fácilmente conculcados. Ese mismo nombre se le dio en las primeras elaboraciones internacionales en este sentido, salidas de la pluma directa del también jurista Paul Otlet (1).

\section{El concepto de Carta de la ONU en Paul Otlet}

Además de los escritos antes citados, conocemos que el maestro de documentalistas elaboró todo un Tratado de la Sociedad de Naciones (Otlet,

Scire. $10: 1$ (en.-jun. 2004) 21-30. 
1917), publicado, según hemos apuntado simultáneamente en Paris y Ginebra cuando ya la Primera Gran Guerra había asolado los campos de Europa (1917). Al hacerlo seguía el pensamiento del Presidente Wilson, quien el 22 de enero de 1917 proclamaba que era necesario crear una fuerza organizada superior a la fuerza de cualquier nación o alianza de naciones y organizar un mundo donde la democracia no sea amenazada de nuevo. La misma idea compartía el inglés Lloyd George, quien el 12 de abril del mismo año escribía: Puedo entrever la paz que llega, no una paz que sea el comienzo de los preparativos para una lucha sin fin, sino una paz sin fin, una paz real, tal cual nuestro mundo no la haya conocido jamás. La Cámara Francesa aprobaba por una mayoría de 453 votos poco después, el 3 de junio de 1917, la siguiente resolución: "Conseguir garantías duraderas de paz y de independencia para los pueblos, grandes o pequeños, dentro de una Organización, ya presente desde ahora: la Sociedad de Naciones" (2).

Pero, ¿dónde habían sido elaboradas estas ideas básicas y fundamentales para la política del siglo XX y de siglos venideros, y de las que nos alimentamos internacionalmente incluso en este momento? El mismo autor nos lo confiesa en el prólogo de la obra comentada: "[...] de un curso impartido en la Escuela de Altos Estudios Sociales, en París, en1916, luego de una exposición general, seguida de debate organizado por la antigua Liga de Naciones [...]". Es evidente, por todo lo expuesto, que Otlet no trabajó sólo; pero no lo es menos que sin su esfuerzo y el de Henry La Fontaine no se hubiera llegado entonces a un alto grado de consenso. Tampoco faltaron en ese empeño los motivos personales, ya que en la Primera Gran Guerra perdía a uno de sus hijos en el frente de Yser.

\section{La intervención directa del documentalista: La Carta de San Francisco y el Proyecto Otletiano}

Ya hemos destacado lo que sin duda alguna constituye una de las características de toda la actuación social y cultural del insigne documentalista - a la par que jurista y sociólogo - belga. Más aún sus propuestas e instituciones llevaban siempre el sello de lo multinacional, porque su sueño era unir culturalmente no sólo a Europa, sino al mundo entero, para que se superasen y olvidasen los odios y discordias seculares (3). Dos de las obras más interesantes e indicativas al respecto, y a las que nos hemos referido más arriba - Tratado de paz general, de octubre de 1914, y Los problemas internacionales y la guerra, de 1916, editadas en pleno conflicto-, hicieron nacer en él, junto a los afanes documentales, la idea de un mundo en paz global. Un nuevo mundo en orden activo que compartiera no sólo recursos, sino sobre todo economía, cultura y ciencia.

Otlet, como Secretario General de la Unión de Asociaciones Internacionales de Bruselas, escribía lo siguiente en la segunda de las obras citadas: "Si la lutte a pu devenir universelle, c'est que les causes en étaient profondes et nombreuses, et

Scire. $10: 1$ (en.-jun. 2004) 21-30. 
une fois déclanchée, c'est une immense révolution qui c'est en voie de s'accomplir dans tous les pays". La primera de esas revoluciones estallaría esperanzadora en un principio - aunque después resultara contraproducente y demoledora para no pocos países europeos, sobre todo los denominados luego del Este- en 1917, en la inmensa Rusia de los zares.

Mas adelante se preguntará el internacionalista: “[...] quelles sont les solutions nouvelles ou renouvelées qui ont été préconisées [...]" y responde a continuación: "[...] l'organisation qu'il serait désirable de donner a la Société des Nations; les conclusions sur l'internationalisme, la sociologie internationale[... ]". Él contemplaba cuatro escenarios después de la inevitable contienda: la conclusión de la Guerra sin Congreso alguno, con imposición de las condiciones a los vencidos; la convocatoria de un Congreso de las potencias beligerantes, que regulara las cuestiones dirimidas en la Guerra, sin dar cabida a los neutrales; la convocatoria de una tercera Conferencia de La Haya, para decidir sobre todas las cuestiones del interés del conjunto de las naciones; y la convocatoria de beligerantes y neutrales para la creación de una Constitución Internacional, la cual dotara de una organización racional a la Sociedad de Naciones, promulgando una carta mundial (Otlet, 1917,p. 482 - 483). En esta última propuesta se volcará el repetido insigne internacionalista como vía más segura de evitar los conflictos, y dirimir las cuestiones que surjan entre los estados. A él debemos atribuir, casi en exclusiva - pues los documentos manuscritos y mecanografiados del Mundaneum lo manifiestan bien a las claras - la paternidad de la idea de la famosa Carta Mundial. Veamos el contenido de algunos de estos valiosos e interesantes documentos, que le ocuparon incluso los ratos de ocio de los años de crisis internacional, y que él departía en charlas y coloquios, así como por carta $\mathrm{y}$ en diversas publicaciones.

\subsection{Los primeros documentos sobre la Sociedad de Naciones y la Paz Mundial}

Por lo indicado hasta aquí queda muy patente la impronta internacionalista de los creadores de la moderna Documentación. Lo que diferencia a esta Ciencia de las tradicionales - Bibliografía, Biblioteconomía y Archivística- es sin duda una nueva visión social de la misma Ciencia, extremo éste bien ajeno a las enunciadas como precedentes instrumentales de la propia Documentación. Su misma concepción como tal es fruto de algo que se deriva directamente del positivismo filosófico del que la Documentación toma carta de naturaleza. Los primeros documentalistas no concibieron este nuevo campo del saber como un instrumento aséptico; sino como un catalizador de nuevas ideas que fueran compartidas por todos los humanos, especialmente los dedicados al cultivo del intelecto y de la información. Podríamos incluso afirmar, sin duda alguna, que tanto Henri La Fontaine como Paul Otlet concibieron la Documentación como

Scire. $10: 1$ (en.-jun. 2004) 21-30. 
un instrumento esencial de la Paz anhelada para la Europa contemporánea y para el mundo en general. Con motivo del XX aniversario de INFODOC, creado en el seno de la Universidad Libre de Bruselas para estudiar los problemas de la moderna Documentación, Jean François FLUEG publicó al respecto un interesante artículo que llevaba por título: Le Mudaneum d'Otlet a Internet, une machine à faire la Paix (1998).

Pero veamos con algún detalle las huellas de estas aportaciones documentales hoy entre los papeles semicatalogados de los creadores del Mundaneum. Además de las grandes obras sobre Documentación e Internacionalismo indicadas más arriba, la Unión de Asociaciones Internacionales, por ellos constituida en 1910, editó otra serie de fascículos, y entre ellos el denominado Periodicum Mundaneum que aparece a finales de los años veinte del pasado siglo. La cabecera y los variadísimos contenidos de este peculiar periódico son una mina de datos sobre internacionalismo y Documentación, y en esta revista se documenta el embrión de varias instituciones fundamentales del pasado siglo e incluso del futuro internacional, de las que citaremos las más esenciales al hilo de nuestro trabajo: Instituto Internacional de Bibliografía y Documentación (1895), con tres campos fundamentales - Repertorio Bibliográfico Universal, Clasificación Internacional y Enciclopedia Documental -, Biblioteca Internacional (1907) con fondos de la Asociación de Intelectuales y Asociaciones Internacionales, Unión de Asociaciones Internacionales (1910), Museo Mundial (1910), Universidad Internacional (1920), Palacio Mundial (1920) y Comité de la Ciudad Internacional (1929). El objetivo general de todas estas instituciones, aparece reflejado en uno de sus textos (4):

Objet: Féderer et faire coopérer entrer elles les Associations Internationales, les Sociétés et les Groupes. Organiser a leur intervention et par de méthodes communes la coordination du travail intellectuel. Constituer la Documentation Mondiale en centralisant les publications et les travaux scientifiques. Sur le nom du Mundaeum, réaliser une représentation du Monde, un dépôt commun pour les collections et un foyer pour les Associations. Travailler a l'établissement d'une cité mondiale installée en territoire exterritorialité, et donc le Mundaneum soit l'un d'institutions.

En cualquiera de estos documentos podemos atisbar que las grandes ideas que movían el mundo político y diplomático de aquellas décadas provenían en gran media del Mundaneum. Ese centro era el que alimentaba los grandes congresos y conferencias internacionales, sobre todo las celebradas en Europa. Todos ellos tenían como principio la mente privilegiada y ordenada del mismísimo Paul Otlet.

\subsection{Documentos autógrafos de Paul Otlet sobre la Sociedad de Naciones}

Dado que la Sociedad de Naciones fue un claro precedente de la actual organización de la política y sociedad internacionales, hemos investigado, después de lo que queda expuesto, acerca de la participación directa de Otlet —sin

Scire. $10: 1$ (en.-jun. 2004) 21-30. 
preterir las aportaciones, sin duda importantes, del ya por entonces Premio Nobel de la Paz 1913 Henri La Fontanine - en toda una serie de ambiciosos proyectos internacionales. Dos documentos, entre los miles que conserva el Mundaneum, nos han parecido determinantes al respecto: En primer lugar, el manuscrito catalogado entre 1992 y 1995 por Christian L'Hoest y Paulette Temmerman, intitulado Charte Mondiale Organisant la Société des Nations, fechado el 20 de diciembre de 1916 (5) y en segundo término, una carta autógrafa de 24 de agosto de 1917, escrita desde el manantial Cachat de Evian les Bains (Francia), donde el internacionalista pasaba sus vacaciones aquel verano de 1917.

Entre esas dos etapas, es decir otoño-invierno de 1916 y primavera-verano de 1917, una de sus preocupaciones primordiales, como lo demuestran además las publicaciones contemporáneas ya citadas, fue la elaboración de un proyecto político-social adecuado, para conseguir que Europa - entonces en cruenta Guerrallegara a una paz y a un statu quo determinantes para su futuro. Así, leemos en la página primera del texto manuscrito y repetidamente corregido por el autor:

"Les alliés combattent pour défendre la justice violée, assurer la liberté à toutes les Nations ... et faire triompher la civilisation. (Déclaration concordante et récherée des Ministres de L'Entante) Pour obtenir ce résultat il faut qu'une Charte mondiale proclame un ordre international et crée des organes nécessaires la Société des Nations

Los apuntes y correcciones personales de ambos manuscritos muestran bien a las claras la cuidadosa redacción del proyecto, y las dudas que tuvo que despejar para llegar a un texto que luego resultara aceptable por todos, tanto para la Sociedad de Naciones, como para la futura ONU. En la segunda página del primero de los documentos aludidos, y al realizar la introducción al mismo texto con el título Conception Général de la Société des Nations, su autor acota entre corchetes el párrafo siguiente: "Traduire ici les p. 422 - 427 des Problèmes Internationaux et la Guerre." Luego elabora un cuidado Prefacio, cuyo proemio no dudamos en transcribir: "[interlineado] Essai de règlement possible après la Guerre". Y continúa: "Il n'y a que deux manières d'envisager le règlement qui suive a cette Guerre: Ou bien il interviendra un Traité par lequel le vainqueur imposera à son adversaire un cumule de conditions particulières inspirées de ses seuls intérêts [...] ou bien, conscients des progrès accomplies dans le Monde, le Traité instaurera un régime nouveau [...]". Al prefacio le siguen los títulos y artículos pertinentes; luego de haber trabajosa y detalladamente redactado el proyecto, Otlet lo dactilografía, y lo corregirá de nuevo en mayo de 1917.

Una vez pensado, redactado y preparado el proyecto, Otlet, de su puño y letra, escribe a su editor de París - desde su merecido descanso- y en la fecha indicada:

Monsieurs: Je viens d'achever un livre sur 'La Constitution Internationale de la Société des Nations'. C'est une Constitution Originale au grand problème actuelle-

Scire. $10: 1$ (en.-jun. 2004) 21-30. 
ment disenté. J'y aborde les difficultés du front, en cherchant à formuler et à commenter un projet complet de Charte que reproduit les parties existantes du droit international et les complète par les propositions présentés depuis la guerre. L'étude est donc d'un ordre politique et juridique. Elle comporterait environ 200 pages de texte.

A continuación viene la propuesta de edición a la editorial parisina: "Je viens vous demander si vous voulez être les éditeurs de mon manuscrit que je vous ferais parvenir à examen si votre réponse de principe était favorable". (Damos casi todo el contenido de la misiva, para que de este modo, quede bien patente la impagable contribución del jurista, sociólogo y documentalista belga a la causa de la Paz entre los pueblos; cuando, como hemos afirmado más arriba, éstos se desangraban en un conflicto de más de tres años.) Y prosigue:

Des études d'ensemble sur le nouveau droit international vont être nécessaires après trois années de guerre. Les milieux français, anglais et Belges s'en préoccupent beaucoup. Une Ligue pour la Société des Nations a été fondée à Paris; une autre à Londres. Je rappelle qu'en 1916 j’ai publié un ouvrage intitulé «Les Problèmes Internationales de la Guerre» d'environ 500 pages que a reçu bon accueil.

A continuación da detalles de las características de la obra y de cómo desea tener presta la edición antes de reintegrarse a las obligaciones normales:

L'ouvrage nouveau devrait être imprimé de suite enfin d'être prêt dès le retour aux travaux régulières. Comptant rester encore quelques temps à Evian, j'en pourrais corriger ici très rapidement les épreuves. Le manuscrit recopié à la machine est en bonne forme.

Al final, Paul Otlet rubrica su texto e introduce una postdata; e indica que, si se diera la posibilidad de que si alguien viniere de París a Suiza, podría conversar con él al respecto, advirtiéndoles que la obtención del pasaporte les llevaría largo tiempo. En octavo aparte expone la tabla de materias del texto propuesto: i) $\mathrm{La}$ Sociedad de Naciones; ii) La Constitución Internacional, a la que después de un preámbulo dedica cuatro títulos - Miembros de la Sociedad de Naciones, Organos y poderes, Vida interna: Relaciones económicas e intelectuales, y Medidas preparatorias y transitorias.

A vuelta del octavo pone su nombre de nuevo con el título elegido para la obra: La constitution internationale de la société des nations. Y concluye con un texto de la Asamblea francesa de 3 de junio de 1917, que marca el objetivo de su empresa: "Obtenir des garanties durables de paix et d'indépendance pour les Peuples grands et petites, dans une Organisation dès maintenent préparée de la Société des Nations".

Entre este proyecto de 1917 y el rubricado en San Francisco en 1946 hay un evidente contacto de paternidad. Sin embargo su autor no podrá reclamar ésta, ya que fallecería el 9 de diciembre de 1944. El Periodiucum Mundaneum de enero de 1946 (p. 2) se felicitaba de que al fin había sido elegido Mr. Paul Henri 
Spaak como Presidente de la Organización de las Naciones Unidas, y con este motivo los amigos del Palacio Mundial: "[...] se félicitent, ils émettent le voeu de voir la Belgique jouer un rôle actif au sein de l’Organisation des Nations Unies, et le Gouvernement belge accorder tout son appui a l'Office Internationale de Bibliographie et de Documentation et a 1'Union des Associations Internationales au sein du Mundaneum". Al final del folio del mismo Periodicum, con el texto del homenaje a Paul Otlet ante su tumba de Etterbeek, aparece la convocatoria de la Conferencia Internacional de la Federación Internacional de Documentación a celebrar en París en el año 1946.

Queda por tanto bien patente que Documentación e Internacionalismo constituyen un binomio inseparable para entender la ingente obra de aquel grupo de pioneros encabezados por Paul Marie Ghislain Otlel y Henri La Fontaine.

\section{Notas}

(1) Paul Otlet fue un eminente jurista, además de parlamentario, bibliógrafo y documentalista, doctorado en Derecho por la Universidad de París y discípulo del ilustre jurista belga Edmond Picard, creador del Journal des Tribunaux y autor de Les Pandectes Belges.

(2) Los textos están recogidos del proemio de la obra citada más arriba (Otlet, 1917).

(3) Este particular nos ocupará en investigaciones sucesivas, ya que hemos encontrado en los archivos del Mundaneum, en Mons, documentos irrefutables de la vertiente europeista de Paul Otlet, hasta el punto de concebir toda una estructura que incluye la nacionalidad europea, para los distintos estados de aquellas primeras décadas del siglo XX.

(4) Texto a pié de página del n ${ }^{\circ}$ 144, fasc. 38, nov. 1937 del Periodicum Mundaneum.

(5) Mundaneum: Dossier 220, 11 y 15.

\section{Referencias}

Charte des Nations unies, statut, reglement et autres textes constitutionnels. $2^{\text {a }}$ ed. Leyde: Société d'Editions, 1947.

Cot, Jean Peirre; Pellet, Alain (1991). La carta de las Naciones Unidas. New York: Ed. NN.UU, 1991. $2^{\text {a }}$ ed. Prólogo de Javier Pérez de Cuellar.

Flueg, Jean Fraçois (1998). Le Mundaneum d'Otlet à Internet... En Roelants -Abraham, Josiane (ed.): Information et documentation: du reel au virtuel. Commémoration du XXième. anniversaire de la Section INFODOC. Bruxelles: Univ. Libre, 1998. 105115.

La Fontaine, Henri; Paul Otlet (1912). La Vie Internationale et l'Effort pour son Organisation. // La Vie Internationale. 1 (1912) 9-34.

Mundaneum. Mons: Ministère de la Communauté française de Belgique, [s. f.]. URL: $<$ http://www.mundaneum.com/>.

Scire. $10: 1$ (en.-jun. 2004) 21-30. 
Otlet, Paul (1909). L'Organisation Internationale et les Associations Internacionales. // Annuaire de la Vie Internationale, 1908- 1909. 2e série, t. 1; Bruxelles: Office Central des Institutions Internationals, 1909. 29-166; También publicado como: Report No. 1; Congrès des Associations Internationales, Bruxelles, 1910 (Bruxelles: n.d. [1909]). $138 \mathrm{p}$.

Otlet, Paul (1914). La Fin de la Guerre: traité du paix général basé sur une charte mondiale déclarant des Droits de 1'Humanité et organisant la Confédération des Etats: extrait de la Vie Internationale, numéro de la Guerre. Bruxelles: L’Union des Associations Internationales, 1914. 159 p. (Publication No. 86).

Otlet, Paul (1916). Les Problèmes Internationaux et la Guerre: tableau des conditions et solutions nouvelles de l'économie, du droit et de la politique. Genève: Librarie Kundig; and Paris: Rousseau et Cie, 1916. 501 p. (UIA Publication No. 50).

Otlet, Paul (1917). Constitution Mondiales de la Societé des Nations: Le Nouveau Droit des Gents. Genève, Ed. ATAR S.A. et Paris, Ed. G. Crès \& Cie., 1917.

Periodicum Mundaneum: Publicaction de L'Union des Associations Internationales. Bruxelles, Mundaneum. Les Institutions du Palais Mondial. Publication trimestrelle avec fascicules intercalaires.

Quintero, Fernando (1995). The typographer who set History. // Berkeleyan: a newspaper for faculty and staff of the University of California at Berkeley. 23:35 (May 17-23, 1995) 1,8 .

Simma, Bruno: The charter of the United Nations. New York: Brokelmann and Rhode eds. 1995.

Union of International Associations (1928). Mundaneum; le centre mondial, scientifique, documentaire et éducatif au service des Associations internationales, qu'il est proposé d'établier à Genève [...]. [Bruxelles, 1928]. (Union des associations internationales; 128). 